\title{
Radiation in the neighbourhood of a double layer
}

\author{
R. Pottelette ${ }^{1}$, M. Berthomier ${ }^{1}$, and J. Pickett ${ }^{2}$ \\ ${ }^{1}$ LPP-CNRS/INSU, 4 avenue de Neptune, 94107 Saint-Maur-des-Fossés, France \\ ${ }^{2}$ Department of Physics and Astronomy, The University of Iowa, Iowa City, IA, USA
}

Correspondence to: R. Pottelette (raymond.pottelette@lpp.polytechnique.fr)

Received: 5 September 2013 - Revised: 5 May 2014 - Accepted: 19 May 2014 - Published: 25 June 2014

\begin{abstract}
In the auroral kilometric radiation (AKR) source region, acceleration layers narrow in altitude and associated with parallel field-aligned potential drops of several $\mathrm{kV}$ can be identified by using both particles and wave-field high time-resolution measurements from the Fast Auroral SnapshoT explorer spacecraft (FAST). These so-called double layers (DLs) are recorded around density enhancements in the auroral cavity, where the enhancement can be at the edge of the cavity or even within the cavity at a small scale. Once immersed in the plasma, DLs necessarily accelerate particles along the magnetic field lines, thereby generating locally strong turbulent processes leading to the formation of nonlinear phase space holes. The FAST data reveal the asymmetric character of the turbulence: the regions located on the highpotential side of the DLs are characterized by the presence of electron holes, while on the low-potential side, ion holes are recorded. The existence of these nonlinear phase space holes may affect the AKR radiation pattern in the neighbourhood of a DL where the electron distribution function is drastically different from a horseshoe shape. We present some observations which illustrate the systematic generation of elementary radiation events occurring significantly above the local electron gyrofrequency in the presence of electron holes. These fine-scale AKR radiators are associated with a local electron distribution which presents a pronounced beam-like shape.
\end{abstract}

Keywords. Space plasma physics (radiation processes)

\section{Introduction}

The present communication is devoted to the study of some strong turbulent processes taking place in the Earth's auroral region in connection with particle acceleration and radiation generation. We use data from the Fast Auroral SnapshoT explorer spacecraft (FAST) which, with its still unrivalled high time-resolution instrumentation and high telemetry rate, provide ample details concerning the complex plasma processes involved in the centre of the auroral acceleration region. This region is marked by the transition layer from the hot tenuous magnetospheric plasma to the cold and dense ionospheric plasma. In this paper we concentrate on the study of the physical processes occurring in the upward current region, i.e. the region that is associated with the visible aurora. The FAST observations have demonstrated unambiguously that parallel potential drops, supported by nonlinear features identified as double layers (DLs), constitute the source of auroral particle acceleration (Carlson et al., 1998). These DLs accelerate the precipitating magnetospheric electrons towards the ionosphere, where they cause the aurora and, vice versa, they accelerate the cold ionospheric ion population into an energetic, though cool upward ion beam.

In the first part of the paper we highlight the role of transient nonlinear structures in the basic microscopic processes regulating the cold-hot plasma interactions in the auroral region. The DL's characteristics are derived by using high time-resolution measurements from the FAST spacecraft for both particles and waves-fields. These measurements show that the DLs are recorded in regions of localized enhanced density structures. The turbulence generated by these DLs is asymmetric: electron holes (EHs) are recorded on the highpotential side of these nonlinear structures while ion holes (IHs) are detected on the low-potential side.

The second part of the paper is devoted to the study of auroral kilometric radiation (AKR) generation in the regions located in the neighbourhood of DLs, i.e in those regions where the parallel electric fields are sited. Being a direct consequence of the parallel acceleration processes, the radiation contains fundamental information on their characteristic spatial and temporal scales (Gurnett and Anderson, 
1981; Pottelette et al., 2001). At large distances from a DL, the FAST observations have shown that the radiation results from an unstable electron "horseshoe" distribution which confirmed many of the fundamental elements of the electroncyclotron maser mechanism (Pritchett, 1984; Pritchett et al., 2002; Ergun et al., 2000). Such a distribution is generated in the presence of an upward-directed electric field that accelerates plasma sheet electrons downward to form a beam-like distribution. When these electrons move down the field lines into increasing magnetic field strength, conservation of their first adiabatic invariant causes their pitch angle to increase as a consequence of the mirror force. In these circumstances, the energetic electron distribution develops a peak at large pitch angles. This produces an extended region on the electron distribution function where $\partial F_{\mathrm{e}}\left(v_{/ /}, v_{\perp}\right) / \partial v_{\perp}>0$ provides the free energy for AKR generation. The emission is generated in the $\mathrm{X}$-mode via the electron-cyclotron maser instability and occurs at frequencies below the nonrelativistic electron gyrofrequency $f_{\text {ce }}$. Moreover, the radio waves are emitted very close to the direction perpendicular to the magnetic field.

One of the main drawbacks regarding AKR generation from a horseshoe distribution lies in the fact that the growth rate becomes significant only when this distribution is fully developed, i.e far away from the location where the electrons have been accelerated by the DLs. Accordingly, the comparably simple picture of AKR generation solely by the global horseshoe distribution might be incomplete because one has to face the unusual situation in which the radiation generated by the electrons becomes more powerful as these electrons move away from the place where they have been accelerated.

The satellite measurements indicate that the AKR frequency spectrum mostly consists of fine spectral features, which are extremely narrow in bandwidth and drift up and down in frequency. These observations provide ample reason for asking whether the presence of nonlinear phase space hole structures generated near DLs could contribute to the production of the observed spectral features. In this case, the fine structured AKR features would result from the motion of phase space holes through space, with the frequency drift mapping the variation of the electron gyrofrequency along the magnetic flux tubes to which they are confined. Since the pioneering work of Pottelette et al. (2001), the radiation from nonlinear phase space holes as a possible mechanism for explaining AKR fine frequency structure generation has been extensively studied (Pottelette et al., 2003; Pottelette and Treumann, 2005; Mutel et al., 2006; Treumann et al., 2011). In the present paper we focus on the analysis of the FAST data obtained in the region located on the highpotential side of a DL. This region is filled with electron holes (EHs) and the electron distribution function is drastically different from a horseshoe. In the neighbourhood of such a DL, the FAST plasma wave tracker detects AKR fine frequency structures generated significantly above the local electron gyrofrequency.
The formation of EHs and their associated radiation mechanism are highly nonlinear and cannot be accessed by a perturbation approach. The only way of investigating such processes is through numerical simulations. However, it was recently demonstrated using a heuristic approach that EHs have the capacity to emit band-limited radiation in good agreement with the observations, but that in the upward current region, the growth rate of a single EH is too small for reproducing the observed fine-structure flux (Treumann et al., 2011). The downward-current region has the advantage of hosting much higher electron densities than in the upwardcurrent region, which significantly increases the value of the growth rate. It was then suggested that, in the downwardcurrent region, EHs are capable of producing the AKR fine frequency structures (Treumann et al., 2012). We return to these conclusions in the light of the analysis of the experimental results obtained by the FAST spacecraft in the heart of the upward-current acceleration region. We show that the observed AKR spectral features can be generated in localized enhanced density structures recorded in the vicinity of DLs.

\section{Turbulence generated in the neighborhood of a double layer}

The observations, recorded in the upward-current region of the aurora, reveal that parallel electric fields take the form of strong DLs near the bottom of the acceleration region (Mozer and Hull, 2001; Ergun et al., 2002). Numerical simulations have shown the character of the turbulence generated in the neighbourhood of such DLs (Singh, 2000; Newman et al., 2001; Goldman et al., 2003). On the high-potential side EHs are generated and move earthward at a speed comparable to the accelerated electrons. On the low-potential side, the DLs release ion holes which move anti-earthward with a speed comparable to the accelerated ion beam. Figure 1 illustrates a satellite-crossing of such a turbulent acceleration layer associated with an anti-earthward directed parallel electric field. As a first simple schematic depiction, the amount of parallel energy $e \Delta \Phi / /$ gained by the anti-earthward directed ion beams on the low-potential side of the DL must be equal to the amount of parallel energy acquired by the earthward propagating electron beams on the high-potential side. This schematic interpretation is obviously oversimplified because in the auroral acceleration region the measured DLs are usually oriented at an oblique angle to the magnetic field in such a way that the perpendicular electric field is much larger than the parallel electric field. Consequently, the ions which are not strongly magnetized can experience a significant polarization drift as they transit the DL. A non-negligible portion of their energy can go into the EXB drift. This is not the case for the electrons which are strongly magnetized, so the electron path is guided by the magnetic field.

In the following we choose FAST orbit 1761 as a text book example to illustrate the crossing of a localized DL. 


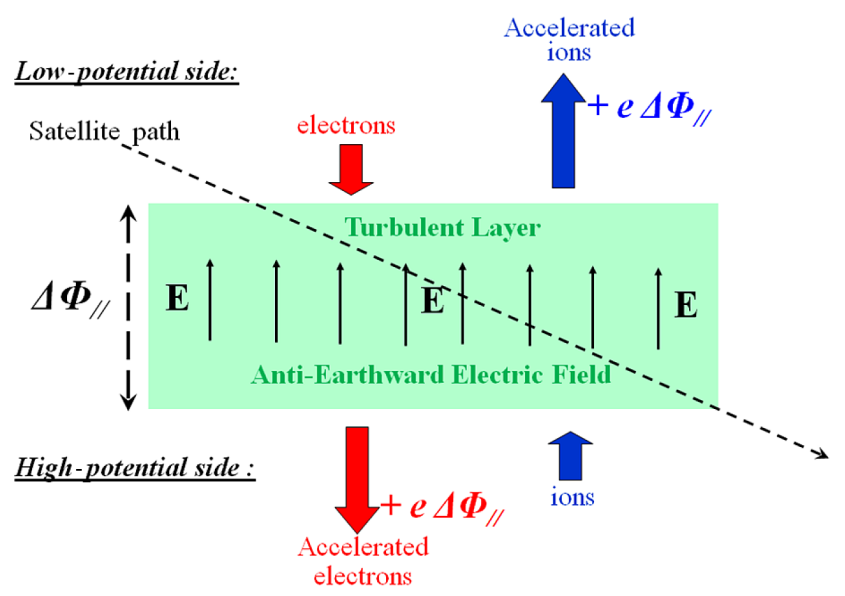

Figure 1. Idealized representation of the field-aligned potential structure in the upward-current region showing the spacecraft path across the parallel electric field layer. When electrons pass across the layer from above they become accelerated while ions are accelerated by the same amount when passing from below.

The FAST spacecraft was on a poleward pass of Earth at an invariant latitude of $\sim 68^{\circ}$ and an altitude of $4150 \mathrm{~km}$ close to apogee. As shown in Fig. 2, we note that the spacecraft approaches the DL from the low-potential side. The parallel energy of the anti-earthward propagating ions drops from $8 \mathrm{keV}$ to a few $\mathrm{eV}$ in $\sim 2.5 \mathrm{~s}$, while during the same amount of time the parallel energy of the earthward propagating electrons increases continuously from 4 to $12 \mathrm{keV}$. This fact provides strong support for crossing a localized potential ramp of $\sim 8 \mathrm{keV}$ amplitude parallel to the magnetic field. This is confirmed well by the simultaneous presence of a large amplitude DC perpendicular electric field $E_{\perp}\left(\sim 1.5 \mathrm{~V} \mathrm{~m}^{-1}\right)$ associated with an anti-earthward directed parallel DC electric field $E_{/ /}$of $\sim 0.3 \mathrm{~V} \mathrm{~m}^{-1}$. Previous observations of this kind have already been published (Ergun et al., 1998, 2002) showing that low-altitude DLs are recorded at the boundary between the auroral cavity and the ionosphere.

In the present case the recorded $8 \mathrm{kV}$ potential drop associated with a $0.3 \mathrm{~V} \mathrm{~m}^{-1}$ amplitude of the parallel electric field implies that FAST was crossing a turbulent accelerating layer with a parallel extension of $\sim 25 \mathrm{~km}$. Note that the event plotted in Fig. 3 does not completely fit the ideal model of a planar, oblique DL since the parallel $E_{/ /}$and perpendicular $E_{\perp}$ electric fields do not have a constant ratio. The strength of the local magnetic field $B \sim 14000 \mathrm{nT}$ together with the recorded amplitude $E_{\perp} \sim 1.5 \mathrm{~V} \mathrm{~m}^{-1}$ produce a convection drift $V_{\mathrm{D}} \sim 100 \mathrm{~km} \mathrm{~s}^{-1}$. So, the perpendicular energy that an $\mathrm{H}^{+}$ion gains as it traverses the DL is about $50 \mathrm{eV}$. This is supported by the measurement of low-energy $(<100 \mathrm{eV})$ ions at pitch angles close to $90^{\circ}$ inside the DL (data not shown). Electrons which are strongly magnetized respond only to the parallel electric field.

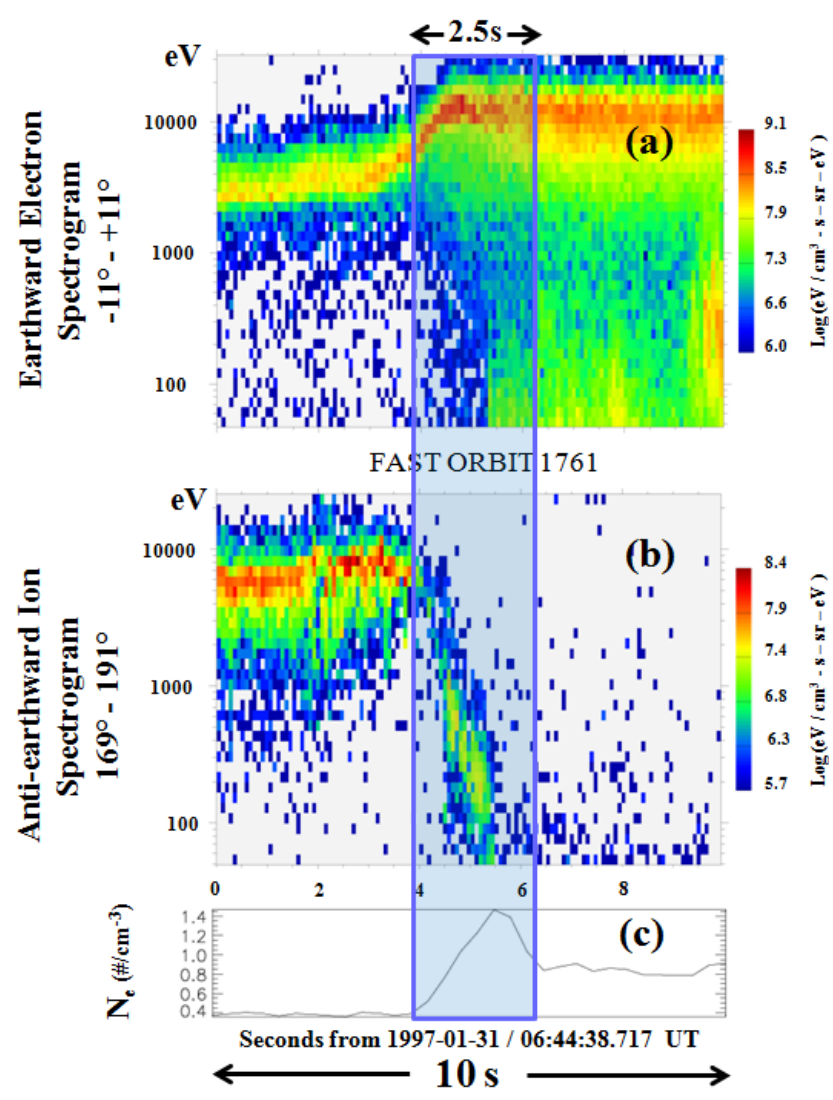

Figure 2. Earthward electron (a) and anti-earthward ion (b) energy fluxes associated with a crossing of a localized double layer. Due to experimental constraints the angular resolution is $\pm 11^{\circ}$. The spacecraft approaches the DL from the low-potential side. The amount of parallel energy lost by the ions is equal to the amount of energy gained by the electrons: $\sim 8 \mathrm{keV}$. The event takes place inside a density enhancement characterized by a large density jump (c). The blue shaded region corresponds to the crossing of the parallel electric field illustrated in Fig. 3.

It is important to point out that this event took place inside a localized density enhancement characterized by a density jump from 0.4 to $1.4 \mathrm{~cm}^{-3}$ (see Fig. 2c). Measuring electron number density from an electrostatic analyzer is difficult, especially at low energies, where spacecraft photoelectrons often dominate the electron flux. The density plotted in Fig. 2c is determined from the electron electrostatic analyzer data above $40 \mathrm{eV}$ in order to eliminate the contribution of photoelectrons. However, the plasma density can also be estimated from the plasma wave cut-off (Ergun et al., 1998; Strangeway et al., 1998). In the present case, the accuracy of both measurements agrees to within about $15 \%$ in the density cavity, whereas the peak value of the density appears to be underestimated by the electrostatic analyzer by about a factor 3 . This indicates that ionospheric electrons have a significant population at energies less than $40 \mathrm{eV}$. Both measurements show the presence of a well-defined enhanced localized density structure regardless of the exact magnitude of the enhancement. 


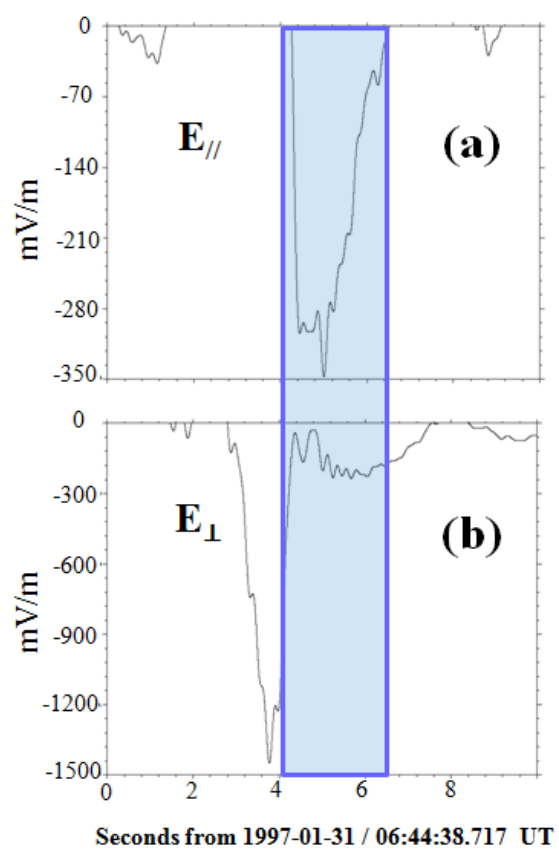

Figure 3. Parallel (a) and perpendicular (b) components of the DC electric field measured at the same time as the electron and ion energy fluxes. The large unipolar negative excursion of the parallel electric field (blue shaded region) indicates the presence of a field aligned potential difference.

Figure 4 reveals, as a function of time, the fluctuations of the $32 \mathrm{kHz}$ parallel electric field waveform recorded in the neighbourhood of a DL whose temporal extension corresponds to the blue-shaded region. As expected, bipolar electric field structures are detected on the high-potential side of the DL; they illustrate the presence of EHs with time spans of about $150 \mu$ s and amplitudes of $\sim 150 \mathrm{mV} \mathrm{m}^{-1}$. These nonlinear structures are positively charged and are thus associated with radial diverging electric fields. All the measured EHs have a positive polarity followed by a negative one (see Fig. 4b) indicating that they are travelling earthward; their velocity should be of the order of the drift velocity of the associated electron beam (Goldman et al., 2003) but cannot be directly determined due to experimental constraints in the present case. On the low-potential side of the DL, large amplitude $\left(\sim 60 \mathrm{mV} \mathrm{m}^{-1}\right)$ electric field structures are also recorded. From their polarity they can be safely identified as anti-earthward moving ion holes. As compared to the EHs, they are detected during a much longer time period ( $\Delta t \sim 5 \mathrm{~ms}$ ), indicating that they have a much lower velocity.

In short the following observations are all in agreement with the crossing of a localized DL by the FAST spacecraft during orbit 1761:

- From the particle measurements it appears that the amount of energy gained by the anti-earthward-directed ion beam on the low-potential side of the DL matches

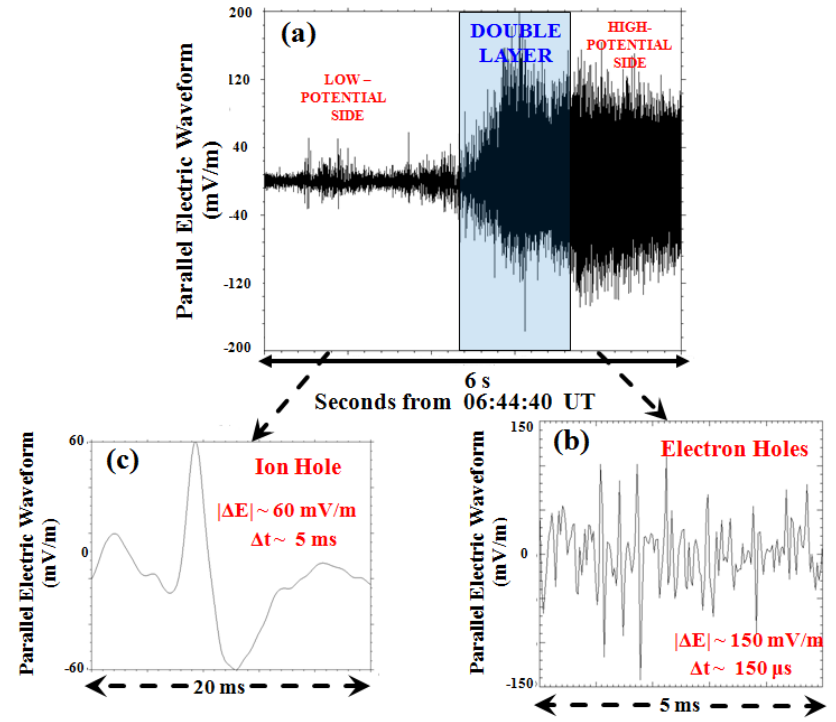

Figure 4. Fluctuations observed in the $32 \mathrm{kHz}$ quasi-parallel electric field waveforms measured by FAST in the neighbourhood of a DL. The temporal extension of the anti-earthward parallel electric field corresponds to the blue shaded region (a). From 06:44:44.713 UT bipolar electric field structures are detected on the high-potential side of the DL (b); they characterize the presence of EHs which have time spans of about $150 \mu \mathrm{s}$ with amplitudes of $\sim 150 \mathrm{mV} \mathrm{m}^{-1}$. On the low-potential side of the DL large amplitude $\left(\sim 60 \mathrm{mV} \mathrm{m}^{-1}\right)$ electric field structures (ion holes) are also recorded at 06:44:40.630 UT; they have time spans of $\sim 5 \mathrm{~ms}(\mathbf{c})$.

the amount of energy gained by the earthward-directed electron beam on the high-potential side.

- The DC measurements show that the particle acceleration takes place in the region where an anti-earthwarddirected parallel electric field is present.

- In agreement with numerical simulations, the analysis of the turbulence generated in the neighbourhood of the DL reveals the presence of ion and electron holes.

On the high-potential side of the low-altitude DLs, the majority of the ionospheric electrons are reflected by the antiearthward-directed parallel electric field and this leads to locally enhanced density structures. It is worth mentioning that similar results are also recorded by the Cluster spacecraft in the heart of the acceleration region (A. Masson, personal communication, 2013). Therefore, the characteristics of the radiation emitted by the accelerated electrons in the vicinity of a DL might be quite different from those recorded inside the density cavity where a nearly complete evacuation of the plasma has taken place. 


\section{FAST ORBIT 1761}
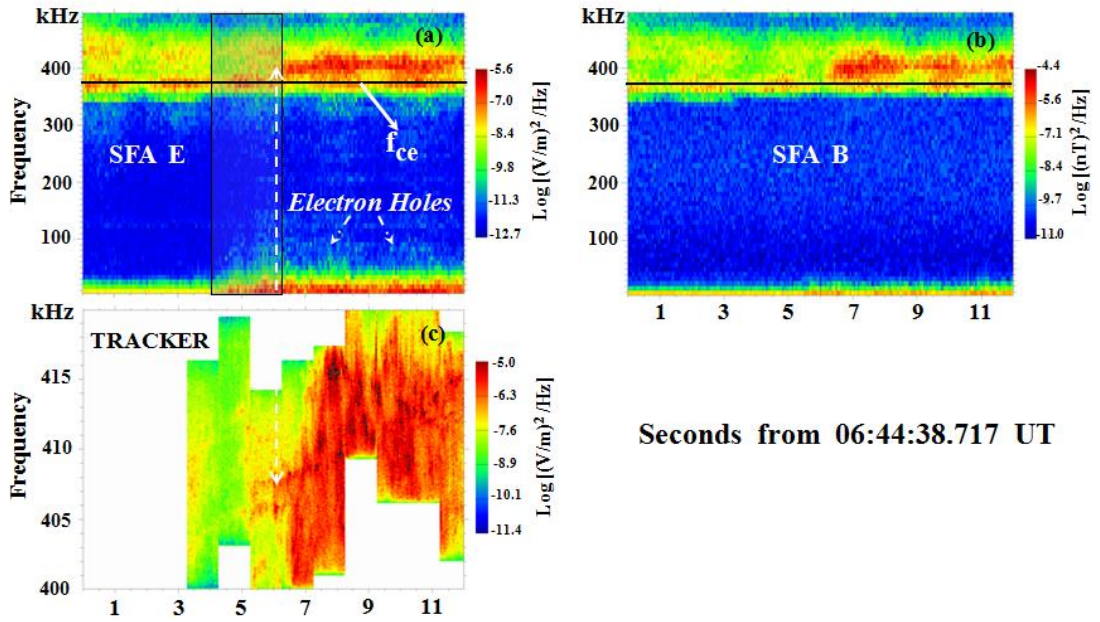

Seconds from 06:44:38.717 UT

Figure 5. Electric (a) and magnetic (b) power frequency spectra measured by the onboard swept frequency analyser (SFA). The frequency spans 0 to $500 \mathrm{kHz}$ with a $4 \mathrm{kHz}$ bandwidth. The shaded blue region again corresponds to the extension of the measured DC parallel electric field. The dark horizontal line is the nonrelativistic electron gyrofrequency $f_{\text {ce }}$. The electric power density (c) provided by the plasma wave tracker reveals the presence of large-amplitude fine spectral AKR features in close relation to the detection of EHs (initial time indicated by the broken white lines). The frequency axis goes from 400 to $420 \mathrm{kHz}$ with a $32 \mathrm{~Hz}$ resolution. Note that the seconds from 06:44:38.717 UT refer to the label for the lower axes.

\section{Radiation generated in the neighbourhood of a double layer}

Figure 5a and $\mathrm{b}$ display the electric and magnetic spectral power density, as a function of frequency and time, generated in the neighbourhood of the DL illustrated in Fig. 3; they show that the high-frequency waves recorded above $f_{\text {ce }}$ are electromagnetic. The magnetic waves in panel $b$ are measured thanks to the onboard search coil. The electric wave power in panel a is derived from a single electric antenna located in the spacecraft spin plane. The satellite is oriented so that the magnetic field lies within $6^{\circ}$ of the spin plane normal in the auroral regions, and the spin period is $5 \mathrm{~s}$. As illustrated in panel a, the AKR emissions recorded at (and slightly below) $f_{\text {ce }}$ show strong spin modulation with a period of $\sim 2.5 \mathrm{~s}$, indicating that the wave electric field is polarized perpendicular to the ambient magnetic field. As previously pointed out by Ergun et al. (2000), these AKR emissions are generated inside the density cavity; they result from the electron-cyclotron maser mechanism. The free energy is provided by the horseshoe electron distribution function measured in these regions, which is displayed in Fig. 6a.

Starting from 06:44:45 UT the most striking feature observed in Fig. 5a and b occurs during the crossing of the high-potential side of the DL: intense AKR emissions are detected some $10 \%$ above the local electron gyrofrequency $\left(f_{\mathrm{ce}} \sim 365 \mathrm{kHz}\right)$ in association with the simultaneous presence of EHs (see Fig. 4). These localized nonlinear structures generate impulsive broadband electric field emissions up to $100 \mathrm{kHz}$ in the swept frequency analyser (SFA) data, indicating that the onboard $32 \mathrm{kHz}$ sampling frequency on the FAST spacecraft may sometime be insufficient to resolve their characteristics entirely. The AKR emissions occurring above $f_{\text {ce }}$ have the following characteristics:

- Their amplitude is about two orders of magnitude larger than the ones occurring around $f_{\text {ce }}$.

- They are associated with an electron distribution showing a pronounced beam-like character as illustrated in Fig. $6 \mathrm{~b}$. The core of the distribution is associated with large $\partial F_{\mathrm{e}}\left(v_{/ /}, v_{\perp}\right) / \partial v_{/ /}>0$ in the vicinity of the peak value of $v_{/ /}$. In addition, for smaller values of $v_{/ /}$, energetic electrons at pitch angles $\theta \sim \pm 45^{\circ}$ are observed, which induce positive perpendicular gradients such that $\partial F_{\mathrm{e}}\left(v_{/ /}, v_{\perp}\right) / \partial v_{\perp}=\frac{\cos \theta}{v} \frac{\partial F_{\mathrm{e}}\left(v_{/}, v_{\perp}\right)}{\partial \theta}$. The existence of these available free energy sources, both in the parallel and perpendicular directions, may induce AKR emissions in a direction oblique with respect tothe magnetic field, as observed. This indicates that these electromagnetic waves have a finite parallel wave vector $k_{/ /}$.

The primary function of the onboard plasma wave tracker (PWT) is to provide fine-frequency resolution spectra of narrow-band emissions. Figure $5 \mathrm{c}$ shows that large amplitude AKR fine frequency structures are detected by the PWT in close relation to the presence of EHs. The fine spectral AKR features consist of a well-defined series of closely spaced absorption and emission bands with several hundred Hertz bandwidths. It is expected that such a structuring results from AKR generation by EHs (Pottelette and Pickett, 

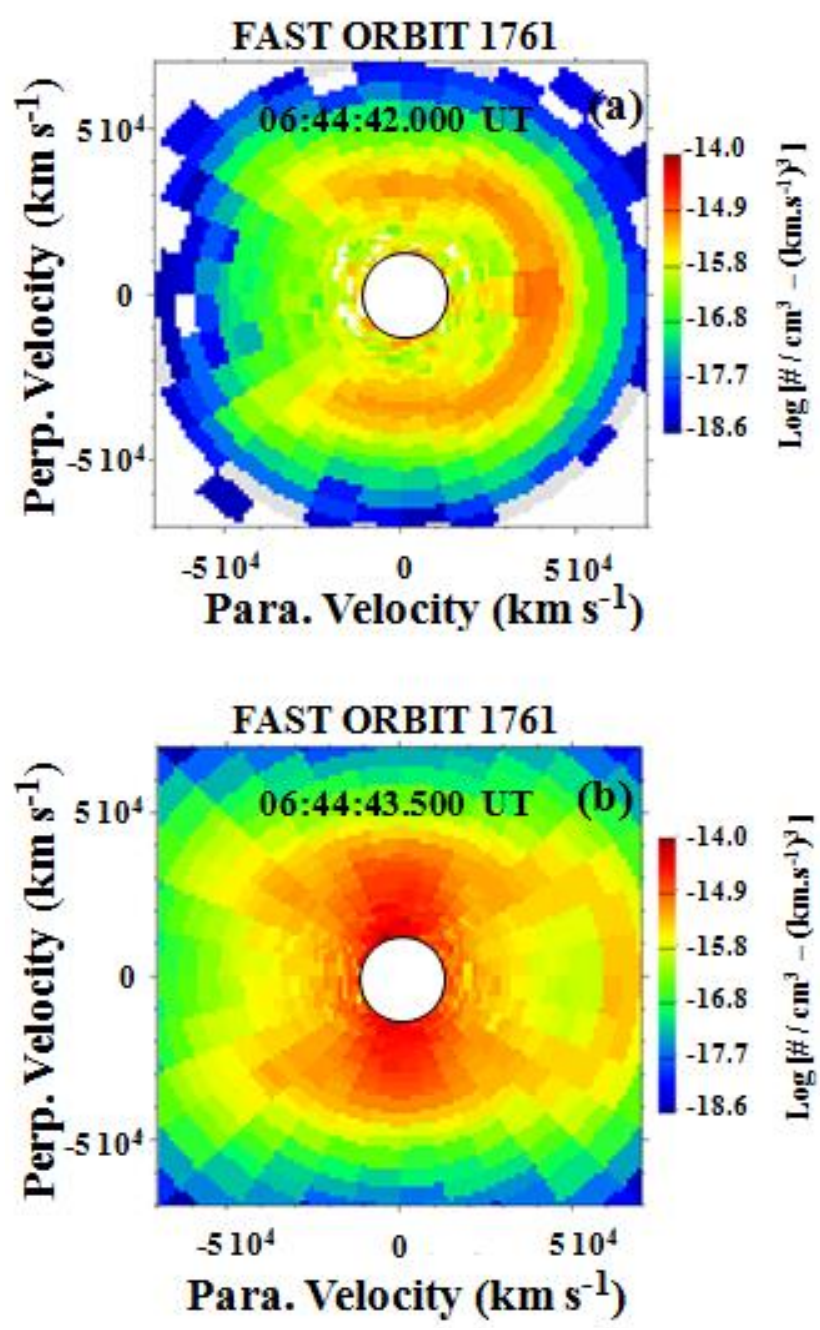

Figure 6. A typical full ring horseshoe distribution of electrons measured inside the density cavity at 06:44:42 UT is shown in (a), while an incomplete horseshoe electron distribution exhibiting a pronounced beam-like character is recorded in the enhanced density region around 06:44:43.500 UT as illustrated in (b). The distributions are plotted in perpendicular, parallel velocity space. Both distributions are averaged over $\sim 0.8 \mathrm{~s}$ and the low-energy electron background has been eliminated in order to remove photoelectrons.

2007; Treumann et al., 2011). The event exhibits predominantly very large, positive frequency drifts $(\mathrm{d} f / \mathrm{d} t>0)$.

Summarizing the analysis of the FAST data from orbit 1761, there is evidence that in the density cavity the free energy source for AKR generation is provided by an electron horseshoe distribution function (Fig. 6a). In agreement with the previous work by Ergun et al. (2000), such AKR emissions are polarized in a direction perpendicular to the magnetic field and occur at or slightly below $f_{\text {ce }}$. In the regions of enhanced density located in the neighbourhood of the DL, the electron distribution exhibits an incomplete ring signature with a pronounced beam-like shape illustrated in Fig. 6b. In these latter regions, the recorded AKR emissions occur significantly above $f_{\text {ce }}$ and are not polarized in a direction perpendicular to the magnetic field.

Observations of this kind are often recorded by the FAST spacecraft when it travels through the acceleration region. Another example taken from orbit 1773 during the crossing of a different AKR source region is displayed in Fig. 7. The spacecraft was travelling poleward at an altitude of $4050 \mathrm{~km}$ and an invariant latitude of $\sim 68^{\circ}$. This orbit has been selected because the PWT measures the characteristics of the high-frequency AKR emissions occurring above $f_{\text {ce }}$ at the same time and in the same frequency range as the SFA. The presence of a localized DL can be derived from the SFA data (Fig. 7a) around 09:26:46 UT when sporadic broadband electrostatic emissions are detected, indicating the presence of EHs. These nonlinear structures are displayed for a $55 \mathrm{~ms}$ period in Fig. 7d. They propagate earthward with amplitudes of $\sim 150 \mathrm{mV} \mathrm{m}^{-1}$ and time spans of $\sim 150 \mu \mathrm{s}$. The fine AKR spectral features recorded by the tracker around this time are illustrated in Fig. 7c. This event covers a frequency range of about $2 \mathrm{kHz}(430-432 \mathrm{kHz})$ but is composed of closely spaced absorption and emission bands with several hundred Hertz bandwidths. These AKR emissions occur at frequencies $20 \%$ above the local electron gyrofrequency $\left(f_{\mathrm{ce}} \sim 360 \mathrm{kHz}\right)$ and exhibit no apparent polarization in a direction perpendicular to the magnetic field. Again, this event took place on the flanks of an enhanced density structure, as illustrated in Fig. 7b. As for orbit 1761, the density has been determined in this figure from the electron electrostatic analyser data above $40 \mathrm{eV}$ in order to eliminate the contribution of photoelectrons. In the present case, using an estimate from the plasma wave cut-off, it appears that the peak value of the density is underestimated by about a factor of 4 .

The electron distribution functions related to this AKR source crossing are displayed in Fig. 8. They are averaged over $\sim 0.8 \mathrm{~s}$. In the density cavity the distribution has an incomplete horseshoe shape (Fig. 8a), while it becomes a partial ring with a pronounced beam-like character in association with the crossing of the DL which generates EHs around 09:26:46 UT (Fig. 8b). In this figure, several distinct regions with large positive parallel velocity gradients can be identified from $v_{/ /} \sim 3.5 \times 10^{4} \mathrm{~km} \mathrm{~s}^{-1}$ to $v_{/ /} \sim 5 \times 10^{4} \mathrm{~km} \mathrm{~s}^{-1}$. This implies that, during the measuring time of the electron distribution function, FAST is crossing a local parallel electric field directed anti-earthward which accelerates the electrons from $\sim 7$ to $\sim 14 \mathrm{keV}$. As in Fig. $6 \mathrm{~b}$, one also notes the perpendicular extents of the distribution around $v_{/ /}=0$ which induce positive perpendicular velocity gradients.

Figure $7 \mathrm{a}$ also shows a very interesting event around 09:26:47.500 UT when weak amplitude broadband emissions covering the entire frequency range are detected. They are generated by a brief incursion of the spacecraft outside the acceleration region. Around this time, the potential moves entirely above the spacecraft as indicated by the sudden recorded large density enhancement. The ions show a conic-like structure while lower-energy electrons 


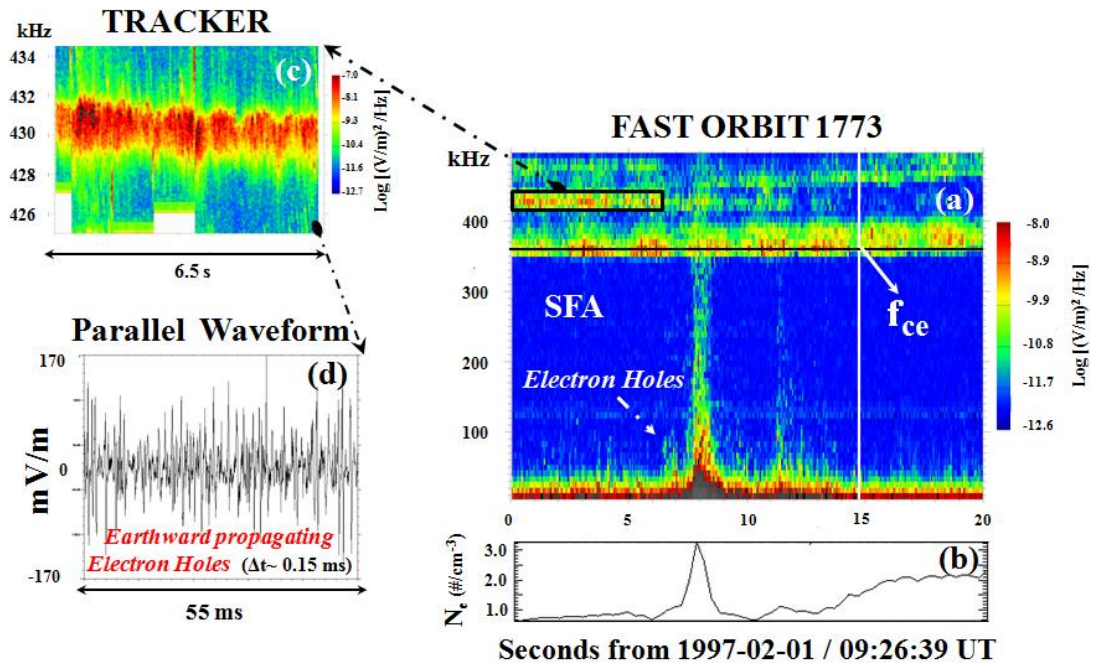

Figure 7. Crossing of an AKR source region during FAST orbit 1773. The presence of a localized DL can be derived from the SFA data (a) around 09:26:46 UT when sporadic broadband electrostatic emissions are detected indicating the presence of EHs. These nonlinear structures are displayed from 09:26:45.769 UT for a $55 \mathrm{~ms}$ period in (d). The fine AKR structures recorded by the tracker during 6.5 s from 09:26:39 UT are illustrated in (c); they cover a frequency range of about $2 \mathrm{kHz}(430-432 \mathrm{kHz})$. Again, this event took place on the flanks of an enhanced density structure as illustrated in (b).

appear within the upgoing source cone (data not shown). After this time earthward-propagating energetic electrons are again detected, consistent with the potential extending to lower altitudes where the FAST spacecraft is located. Another region of sporadic broadband emissions associated with a moderate local density enhancement is also recorded around 09:26:51 UT. Weak transient enhanced AKR emissions above $f_{\text {ce }}$ are detected by the SFA but unfortunately not by the PWT; they are again associated with the presence of EHs (data not shown).

\section{Discussions}

In short, a systematic study of FAST data shows that EHs are ubiquitous in the AKR source region; they are excited at the edge of the density cavity or within small-scale density enhancement structures inside the cavity. At FAST altitudes, EHs are not detected in the cavity itself apart from within the small-scale density structures. These EHs are produced in the neighbourhood of a DL and are associated with an incomplete electron horseshoe distribution function showing a well-pronounced beam-like signature. The analysis reveals that, in close relation with the simultaneous presence of EHs, fine AKR spectral features can be generated at frequencies located significantly above the local $f_{\text {ce }}$. In contrast to the AKR emissions recorded in the density cavity, such AKR fine structures are not polarized in a direction perpendicular to the local magnetic field indicating that they possess a finite parallel wave vector $k_{/ /}$.

One of the fundamental questions to ask is the following: are the AKR emissions recorded in the vicinity of a
DL locally generated or are these emissions beamed to the spacecraft from a small source below the FAST altitude? Assuming a generation at the local $f_{\text {ce }}$ below the spacecraft and dipole magnetic field geometry, we can estimate the distance to the source from the frequency of these emissions. It is $\sim 400 \mathrm{~km}$ for orbit 1761 and $\sim 600 \mathrm{~km}$ for orbit 1773 . Such an explanation cannot be excluded. This would mean that the local presence of EHs inhibits the generation of electromagnetic radiation.

On the other hand, as the AKR emissions recorded significantly above $f_{\text {ce }}$ occur in the immediate vicinity of a DL, it is natural to think that the regions where the electrons are the most strongly accelerated should be the source of intense electromagnetic radiation. For instance, the recorded AKR amplitudes above $f_{\text {ce }}$ reach $\sim 10^{-6}(\mathrm{~V} / \mathrm{m})^{2} \mathrm{~Hz}^{-1}$ for orbit 1761; this is about two orders of magnitude greater than the ones recorded in the cavity (Fig. 5).

As previously mentioned, the electron distribution functions $F_{\mathrm{e}}\left(v_{/ /}, v_{\perp}\right)$ recorded in the vicinity of a DL possess available free energy sources, both in the parallel and perpendicular directions (see Figs. 6b and 8b). This may explain why, in these regions, AKR emissions are generated in a direction oblique with respect to the magnetic field, as observed. However, it does not provide any valuable explanation with regard to the generation of elementary radiation events occurring significantly above $f_{\text {ce }}$. Given the FAST experimental observations, it seems natural to think that EHs have the capacity to emit such band-limited radiation.

The processes involved in AKR generation by EHs have been recently studied theoretically by Treumann et al. (2011). The authors use a heuristic approach and assume 


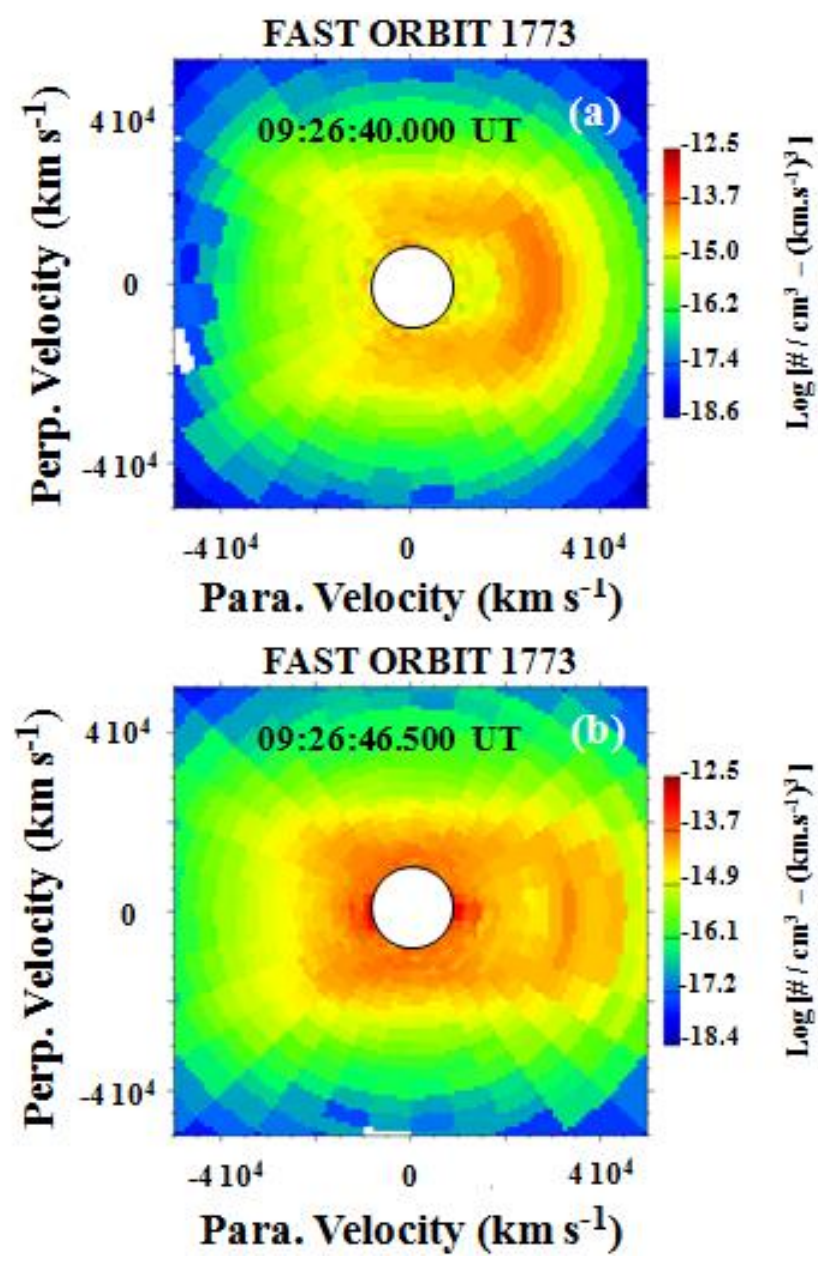

Figure 8. Electron distribution function recorded around 09:26:40 UT in the density cavity (a), incomplete ring distribution with a pronounced beam signature detected around 09:26:46.500 UT during the locally enhanced density structure (b) for the FAST orbit 1773. Both distributions are averaged over $\sim 0.8 \mathrm{~s}$ and the low-energy electron background has been eliminated.

that EHs are evolving inside a horseshoe distribution function, generating significantly large $\partial F_{\mathrm{e}}\left(v_{/ /}, v_{\perp}\right) / \partial v_{\perp}>0$ needed for the electron-cyclotron maser instability. This hypothesis does not fully correspond to the observations, which bring to light that EHs are generated in the regions of localized enhanced density structures by a predominantly beamlike electron distribution function resulting from a local acceleration parallel to the magnetic field. However, some parts of the arguments advanced in this theory provide some clues that EHs could contribute to the generation of radiation.

\subsection{Electron Holes as radiating antennas}

The Debye length in the acceleration region is $\lambda_{\mathrm{D}} \sim 200 \mathrm{~m}$. Assuming a spatial extension $L_{/ /}$of the EH parallel to the magnetic field of a few Debye lengths one gets $L_{/ /} \sim 1 \mathrm{~km}$. Concerning the transverse scale we note that the theory by Berthomier et al. (2003) predicts spherical holes in configuration space in the case where the plasma frequency is small as compared to $f_{\text {ce. }}$. This is in agreement with the observations by Franz et al. (2000). Such a perpendicular size is wellsuited for trapping wavelengths of the order of $\sim 1 \mathrm{~km}$. Thus the EHs can be considered as efficient radiating antennas in the kilometre wavelength range.

In the absence of a magnetic field, EHs have a hole in the electron parallel velocity distribution localized near its velocity $V_{\mathrm{h}}$ in configuration space (Newman et al., 2001). In these circumstances, the numerical simulations show that EHs are very narrow in configuration space while occupying a substantial part of parallel velocity space. The depletion region contains the hot electrons trapped inside the hole. Therefore, in the presence of a magnetic field, the expectation is that the depletion near $V_{\mathrm{h}}$ extends in perpendicular velocity up to the perpendicular thermal velocity of the trapped electrons. To be an important radiation source via the electron-cyclotron maser mechanism, EHs should produce steep positive velocity space gradients on the electron distribution function both in the perpendicular and parallel directions (Treumann et al., 2011). It is important to mention that, thus far, perpendicular velocity space data are unfortunately not represented in numerical magnetized simulations (Oppenheim et al., 2001).

The general weakly relativistic resonance condition for the electron-cyclotron maser instability in terms of the electron velocity components can be written as (cf., e.g., Treumann and Baumjohann, 1997, Chapt. 5, 120-125):

$\left(v_{/ /}-\frac{k_{/ /} c^{2}}{\omega_{\mathrm{ce}}}\right)^{2}+v_{\perp}^{2}=\frac{2 c^{2}}{\omega_{\mathrm{ce}}}\left(\omega_{\mathrm{ce}}-\omega\right)+\frac{k_{/ /}^{2} c^{4}}{\omega_{\mathrm{ce}}^{2}}$,

with $\omega$ the angular wave frequency, $\omega_{\mathrm{ce}}$ the angular electron gyrofrequency, $k_{/ /}$the modulus of the wave vector parallel to the magnetic field and $c$ the speed of light. It describes a resonant circle shifted along the parallel velocity direction. For satisfying the resonance condition, the velocity $V_{\mathrm{h}}$ of the hole must be such that the perpendicular gradients localized at the holes coincide with the resonance contour which implies that

$V_{\mathrm{h}}=\frac{k_{/ / c^{2}}}{\omega_{\mathrm{ce}}}$.

This relation provides a determination of the parallel wave number from the hole velocity and shows that the radiation emitted by an $\mathrm{EH}$ is oblique with respect to the magnetic field, in agreement with the observations previously described.

\subsection{Frequency range and growth rate}

One of the remaining challenges concerning the experimental observations consists of explaining the fact that the AKR 
emissions generated in the vicinity of a DL occur at frequencies sited 10 to $20 \%$ above the local $f_{\text {ce }}$. First, it is worth mentioning that an $\mathrm{EH}$ acting as a radiating antenna selects a wavelength of the order of its linear scale and this impinges on the value of the radiated frequency. As previously mentioned, in the auroral upward-current region, the EH's perpendicular size is well suited for trapping wavelengths of the order of $\sim 1 \mathrm{~km}$. Trapping inside the EHs might be a viable process for keeping the radiation in resonance and amplifying it over the lifetime of the holes. But trapping cannot be complete because the amplification would become excessively large. So, one attractive hypothesis is to assume that the waves are partially trapped and amplified in the EHs and that a fraction of these waves is radiated outside the EHs during their propagation. Since EHs are generated in locally enhanced density structures, the radiated waves can escape because they can propagate freely above the X-mode cut-off in the surrounding regions of decreasing density.

An approximate order of magnitude of the maximum growth rate of the electron-cyclotron maser radiation produced by EHs is given by Treumann et al. (2011). It can be written as

$$
\frac{\gamma}{\omega_{\mathrm{ce}}} \approx \frac{\alpha \pi^{3}}{8} \frac{f_{\mathrm{e}}^{2}}{f_{\mathrm{ce}}^{2}},
$$

with $\alpha=n_{\mathrm{b}} / n_{\mathrm{e}}$ the ratio of the electron beam to the background plasma densities and $f_{\mathrm{e}}$ being the total plasma frequency. Let us take the concrete example of the electron distribution plotted in Fig. $6 \mathrm{~b}$ recorded in the presence of EHs during orbit 1761. The electron beam density is calculated from the electron spectrometer data taking an opening angle of $\pm 11^{\circ}$ (experimental constraints), which yields $n_{\mathrm{b}} \sim 0.05 \mathrm{~cm}^{-3}$. The value of the peak density estimated from the plasma wave cut-off is $n_{\mathrm{e}} \sim 4 \mathrm{~cm}^{-3}$ leading to $f_{\mathrm{e}} \sim 18 \mathrm{kHz}$. For $f_{\text {ce }} \sim 360 \mathrm{kHz}$, we get $\gamma \approx 10^{-3} \omega_{\text {ce. This }}$ is an appreciable growth rate of the same order of magnitude as the one obtained for a global ring horseshoe distribution (Pritchett, 1986; Louarn, 2006).

An additional effect has to be considered. In the proper frame of the hole, emitted frequencies are slightly beneath the local nonrelativistic $f_{\text {ce }}$ (Treumann et al., 2011). In the observer's frame, the change in frequency due to the displacement of the EHs along the magnetic field is described by the relativistic Doppler effect which, for the observed frequency $f$ of the radiation, yields

$f=f_{\mathrm{ce}} \frac{\sqrt{1-\beta_{\mathrm{h}}^{2}}}{1 \pm \beta_{\mathrm{h}}}$,

where $\beta_{\mathrm{h}}=V_{\mathrm{h}} / c$. The sign \pm in the denominator applies to holes either moving away or approaching. The radiation propagates earthward and is emitted obliquely with respect to the magnetic field. In these circumstances, only signals generated by approaching holes are detected in the satellite

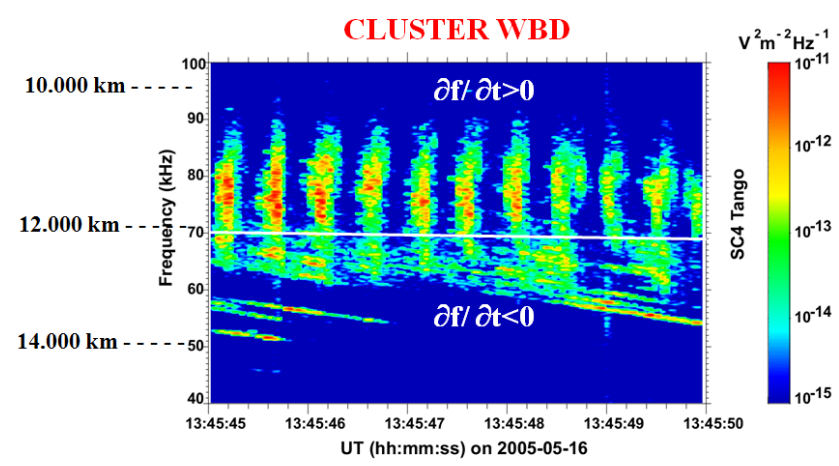

Figure 9. Spectrogram covering a period of $5 \mathrm{~s}$ showing pulsed and striated AKR observed by remote sensing on a Cluster satellite located in the nightside magnetosphere at $14.2 R_{\mathrm{E}}$ and at invariant latitude of $80^{\circ}$. The horizontal white line indicates the value of the local gyrofrequency $f_{\text {ce }} \sim 70 \mathrm{kHz}$ at an altitude of $\sim 12000 \mathrm{~km}$ (adapted from Pottelette and Pickett, 2007).

frame and constitute the small-scale AKR radiators. Assuming an $\mathrm{EH}$ velocity close to the electron drift beam velocity, one gets $V_{\mathrm{h} \sim 4} \sim 10^{4} \mathrm{~km} \mathrm{~s}^{-1}$ (see Fig. 6b), leading to $f \sim 1.14 f_{\text {ce }}$, which is in reasonable agreement with the observations.

\section{Conclusions}

The careful analysis of FAST data reveals that two different AKR sources may coexist at the spacecraft altitudes around $4000 \mathrm{~km}$. They both arise from the electron-maser mechanism:

- In the density cavity, the radiation results from an unstable "horseshoe" electron distribution function. The emission is generated in the X-mode slightly below the nonrelativistic electron $f_{\text {ce }}$. This radio wave is emitted very close to the direction perpendicular to the local magnetic field (Ergun et al., 2000; Pritchett et al., 2002).

- Around density enhancements in the auroral cavity, the free energy leading to AKR fine spectral feature generation may be provided by the presence of EHs. On the electron distribution, they provide steep positive velocity space gradients which are restricted to their edges in the parallel and perpendicular directions. The emission is generated in the $\mathrm{X}$-mode at frequencies located significantly above $f_{\text {ce }}$. It is radiated in a direction oblique with respect to the magnetic field. The electron density being locally enhanced, the growth rate of the electronmaser instability becomes appreciable.

The $\mathrm{EH}$ radiation mechanism investigated in this paper reproduces several features of the fine structure in the AKR. It can explain the frequency drifts with $\mathrm{d} f / \mathrm{d} t>0$ which are illustrated in Figs. 5c and 7c. Unfortunately the induced frequency drifts in these figures are too large to be accurately 
determined given the experimental constraints. It is thus impossible to deduce the spatial radial velocities of the associated structures from FAST data (Pottelette and Pickett, 2007).

Regarding the AKR frequency drifts associated with $\mathrm{d} f / \mathrm{d} t<0$, it was earlier pointed out that such drifts could be generated by isolated parallel electric field structures of tripolar polarity, being interpreted in terms of trains of nested ions and electron holes (Goldman et al., 2003). Such nonlinear structures have indeed been detected on the low-potential side of a DL (Pottelette and Treumann, 2005). Consequently, both ion and electron hole dynamics seem to play a major role in the generation of the subtle, fine frequency structures moving across the radiation spectrum.

This is illustrated well by the data displayed in Fig. 9, which shows a stunning example of AKR fine frequency structures remotely recorded by the Cluster spacecraft in the nightside magnetosphere. The radiation appears to be generated at an altitude of $\sim 12000 \mathrm{~km}$ matching with a local electron gyrofrequency $f_{\text {ce }} \sim 70 \mathrm{kHz}$. The AKR amplitude is modulated at a frequency of $\sim 2.8 \mathrm{~Hz}$, which is in the frequency range of ULF waves. Pulsations at these frequencies are generated by the electromagnetic ion cyclotron instability near the magnetic equator and are called Pc1 waves. The spectrum in Fig. 9 reveals two different types of frequency structures. The first one known as striated AKR (Mutel et al., 2006), is associated with moderate slope frequency drifts $\mathrm{d} f / \mathrm{d} t<0$ and is recorded below $f_{\text {ce }}$ at the low-frequency edge of the spectrum. The second type is associated with an abrupt upward frequency expansion $(\mathrm{d} f / \mathrm{d} t>0)$ above $f_{\text {ce }}$. These observations can be interpreted as a result of a local acceleration by a pulsing DL located at a quasi-steady altitude of $\sim 12000 \mathrm{~km}$. Upstream of this layer, at a frequency smaller than $f_{\text {ce }}$, the usual striated AKR emissions are recorded with a mean slope of $-2.4 \mathrm{kHz} \mathrm{s}^{-1}$, corresponding to a trigger speed of $210 \mathrm{~km} \mathrm{~s}^{-1}$, which matches with anti-earthward propagating ion hole speeds (Bounds et al., 1999). Downstream from the accelerating layer, an abrupt frequency expansion above $f_{\text {ce }}$ is recorded. Again, what is noteworthy, is the presence of a well-defined series of closely spaced absorption and emission bands (separated by several hundred Hertz) which may characterize AKR generation by EHs propagating earthward at high velocity (Pottelette and Pickett, 2007).

This example is very complementary to the FAST observations as it occurs at much higher altitudes at the top level of the auroral acceleration regions where the ring horseshoe electron distribution is not fully developed. It clearly suggests that DLs and phase space holes are crucial elements in the generation of the fine AKR spectral structures. However, there are crucial issues which need to be solved before it is firmly established that such nonlinear localized structures radiate the fine spectral features in the AKR spectrum. The formation of phase space holes and their associated radiation mechanism are highly nonlinear and cannot be accessed by a perturbation approach. These observational results might encourage investigation of such radiating processes through numerical simulations because they could be relevant to other astrophysical radio sources.

Acknowledgements. The FAST mission is a project of the Space Sciences Laboratory of the University of California at Berkeley run under the auspices of NASA. The authors are indebted to C. W. Carlson and R. E. Ergun for providing particle and wave data as well as for some useful discussions. This research has been initiated within the France-Berkeley Program. Work at the University of Iowa was carried out under NASA GSFC Grant No. NNX11AB38G and NNX14AB10G. This paper has benefited from several discussions held during the Auroral Plasma Physics Workshop in Aussois (April 2013, France). Support for the organization of this meeting was provided by grants from the CNRS National Program "Soleil Terre".

Topical Editor L. Blomberg thanks four anonymous referees for their help in evaluating this paper.

\section{References}

Berthomier, M., Pottelette, R., Muschietti, L., Roth, I., and Carlson, C. W.: Scaling of three dimensional electron phase space density holes observed by FAST in the auroral downward current region, Geophys. Res. Lett., 30, 2148-2152, doi:10.1029/2003GL018491, 2003.

Bounds, S. R., Pfaff, R. F., Knowlton, S. F., Mozer, F. S., Temerin, M. A., and Kletzing, C. A. : Solitary structures associated with ion and electron beams near 1 Re altitude, J. Geophys. Res, 104, 28709-28717, 1999.

Carlson, C. W., Pfaff, R. F., and Watzin, G.: Fast Auroral Snapshot (FAST) mission, Geophys. Res. Lett., 25, 2013-2016, doi:10.1029/98GL01592, 1998.

Ergun, R. E., Carlson, C. W., McFadden, J. P., Mozer, F. S., Delory, G. T., Peria, W., Chaston, C. C., Temerin, M., Roth, I., Muschietti, L., Elphic, R., Strangeway, R., Pfaff, R., Cattell, C. A., Klumpar, D., Shelley, E., Peterson, W., Moebius, E., and Kistler, L.: FAST satellite observations of largeamplitude solitary structures, Geophys. Res. Lett., 25, 20412044, doi:10.1029/98GL00636, 1998.

Ergun, R. E., Carlson, C. W., McFadden, J. P., Delory, G. T., Strangeway, R. J., and Pritchett, P. L.: Electron-Cyclotron Maser Driven by Charged-Particle Acceleration from Magnetic Field-aligned Electric Fields, Astrophys J., 538, 456, doi:10.1086/309094, 2000.

Ergun, R. E., Andersson, L., Main, D., Su, Y. J., Newman, D. L., Goldman, M. V., Carlson, C. W., McFadden, J. P., and Mozer, F. S.: Parallel electric fields in the upward current region of the aurora: Numerical solutions, Phys. Plasmas, 9, 3695-3704, doi:10.1063/1.1499121, 2002.

Franz, J. R., Kintner, P. M., Seyler, C. E., Pickett, J. S., and Scudder, J. D.: On the perpendicular scale of electron phase-space holes, Geophys. Res. Lett., 27, 169-173, doi:10.1029/1999GL010733, 2000.

Goldman, M. V., Newman, D. L., and Ergun, R. E.: Phase-space holes due to electron and ion beams accelerated by a current- 
driven potential ramp, Nonlin. Processes Geophys., 10, 37-44, doi:10.5194/npg-10-37-2003, 2003.

Gurnett, D. A. and Anderson, R. R.: The kilometric radio emission spectrum: relationship to auroral acceleration processes, in Physic of auroral arc formation, edited by: Akasofu, S.-I. and Kan, J. R., AGU, Washington D.C., Monogr., 25, 341-350, 1981.

Louarn, P.: Generation of auroral kilometric radiation in bounded source regions, in Geospace Electromagnetic Waves and Radiation, Lecture Notes in Physics (LPN), Vol. 687, Springer-Verlag, Berlin-Heidelberg-New York, 55-86, 2006.

Mozer, F. S. and Hull, A.: Origin and geometry of upward parallel electric fields in the auroral acceleration region, J. Geophys. Res, 106, 5763-5778, doi:10.1029/2000JA900117, 2001.

Mutel, R. L., Menietti, J. D., Christopher, I. W., Gurnett, D. A., and Cook, J. M.: Striated auroral kilometric radiation emission: A remote tracer of ion solitary structures, J. Geophys. Res., 111, A10203, doi:10.1029/2006JA011660, 2006.

Newman, D. L., Goldman, M. V., Ergun, R. E., and Mangeney, A.: Formation of Double Layers and Electron Holes in a Current-Driven Space Plasma, Phys. Rev. Lett., 87, 255001, doi:10.1103/PhysRevLett.87.255001, 2001.

Oppenheim, M. M., Vetoulis, G., Newman, D. L., and Goldman, M. V.: Evolution of electron phase-space holes in 3D, Geophys. Res. Lett., 28, 1891-1894, doi:10.1029/2000GL012383, 2001.

Pottelette, R. and Pickett, J.: Phase space holes and elementary radiation events, Nonlin. Processes Geophys., 14, 735-742, doi:10.5194/npg-14-735-2007, 2007.

Pottelette, R. and Treumann, R. A.: Electron holes in the auroral upward current region, Geophys. Res. Lett., 32, L12104, doi:10.1029/2005GL022547, 2005.

Pottelette, R., Treumann, R. A., and Berthomier, M.: Auroral Plasma Turbulence and the cause of AKR fine structure, J. Geophys. Res., Vol. 106, 8465-8476, doi:10.1029/2000JA000098, 2001.
Pottelette, R., Treumann, R. A., Berthomier, M., and Jasperse, J.: Electrostatic shock properties inferred from AKR fine structure, Nonlin. Processes Geophys., 10, 87-92, doi:10.5194/npg-10-872003, 2003.

Pritchett, P. L.: Relativistic dispersion and the generation of auroral kilometric radiation, Geophys. Res. Lett., 11, 143-146, doi:10.1029/GL011i002p00143, 1984.

Pritchett, P. L.: Electron cyclotron maser instability in relativistic plasma, Phys. Fluids, 29, 229-315, doi:10.1063/1.865492, 1986.

Pritchett, P. L., Strangeway, R. J., Ergun R. E., and Carlson, C. $\mathrm{W}$ : Generation and propagation of cyclotron maser emissions in the finite ARK source cavity, J. Geophys. Res., 107, 1437-1447, doi:10.1029/2002JA009403, 2002.

Singh, N.: Electron holes as a common feature of double layer-driven plasma waves. Geophys. Res. Lett., 27, 927-930, doi:10.1029/1999GL003709, 2000.

Strangeway, R. J., Kepko, L., Elphic, R. C., Carlson, C. W., Ergun, R. E., McFadden, J. P., Peria, W. J., Delory, G. T., Chaston, C. C., Temerin, M., Cattell, C. A., Möbius, E., Kistler, L. M., Klumpar, D. M., Peterson, W. K., Shelley, E. G., and Pfaff, R. F.: FAST observations of VLF waves in the auroral zone: Evidence of very low plasma densities, Geophys. Res. Lett., 25, 2065, doi:10.1029/98GL00664, 1998.

Treumann, R. A. and Baumjohann, W.: Advanced Space Plasma Physics, Imperial College Press, London, 1997.

Treumann, R. A., Baumjohann, W., and Pottelette, R.: Electroncylotron maser radiation from electron holes: upward current region, Ann. Geophys., 29, 1885-1904, doi:10.5194/angeo-291885-2011, 2011.

Treumann, R. A., Baumjohann, W., and Pottelette, R.: Electroncylotron maser radiation from electron holes: downward current region, Ann. Geophys., 30, 119-130, doi:10.5194/angeo-30-1192012, 2012. 